\title{
Study on Collapse Sequence and Critical Rainfall at Nantou 89th County Road in Taiwan
}

\author{
Sung-Chi Hsu', Pei-Ju Hu², Pei-Hsun Tsai \\ ${ }^{1}$ Chaoyang University of Technology \\ 168 Gifong E. Rd., Wufong, Taichung, Taiwan \\ schsu@cyut.edu.tw; jal8304@gmail.com \\ ${ }^{2}$ Chaoyang University of Technology \\ 168 Gifong E. Rd., Wufong, Taichung, Taiwan \\ phtsai@cyut.edu.tw
}

\section{Extended Abstract}

Taiwan's rainfall is concentrated in the plum rain season and typhoons from May to October every year, often with a large amount of water vapor, causing concentrated rainfall in the mountains. Due to climate change, the chances of extremely heavy rain have increased and caused the probability of slope damages, resulting in disasters such as collapses, slope failures and mudslides [1-4]. The area of 89th Country Road of Ren-ai Township, Nantou County was selected for the present study. The area is important because it is the main road to Lishan Scenic Area and many tribes live along the road. Because of fragile geology and friable topography, it easily causes the caving in of roadbed. Several locations had collapsed and caused the closure of the road. This study discusses the history of damage due to critical rainfall of $12.3 \mathrm{~K}$ and $22.53 \mathrm{~K}$ on the 89 th Country Road. The aerial photos of an exposed range of $12.3 \mathrm{~K}$ and $22.53 \mathrm{~K}$ from 2001 to 2017 were collected to carry out the image overlay and comparison. Also, we used previous destruction events for comparison and sorting out the failure processes and mechanisms. It was found that the damage of $12.3 \mathrm{~K}$ initiated from an eroded ditch on the lower slope of the road. The gully caused retrogressive erosion of downhill slope and induced the collapse of roadbed and slippage of the slope. The initial damage of $22.53 \mathrm{~K}$ commenced from the right side of the downstream. The erosion ditch caused the collapse and slippage due to lateral and headward erosion. After that, the erosion ditch gradually continued developing upward to the bottom of the road and affected the road to be diverted to the upper slope. The topographic accumulation-scouring map of $12.3 \mathrm{~K}$ during 2008-2010 showed that severed erosion and sliding events had occurred at downslope and upslope, especially for the downslope areas. The difference of eroded thickness was more than $15 \mathrm{~m}$. The accumulation-scouring map of 20102012 showed that severe erosion had occurred around the ditch. The difference of eroded thickness was more than $20 \mathrm{~m}$. Aerial photo of $22.53 \mathrm{~K}$ during $2008-2010$ showed that the left side of the gully ditch was washed sharply and the difference of elevation thickness was more than $40 \mathrm{~m}$. The slopes above and below the road suffered severe sliding which caused the difference of elevation more than $15 \mathrm{~m}$ between 2010 and 2012.

The research also compares the major rain incidents, which caused failure or non-failure of a slope, at Nantou 89th Country Road. Several decision charts related to rainfall characteristics have been drawn. Based on this study, the obtained critical rainfall is about $240 \mathrm{~mm}$ for these two locations. The I-T type and I-R type rain warning mode are recommended for the decision of evacuation and closure of the road in the area.

The results of this study could be used as a reference for disaster prevention and evacuation in the future.

\section{References}

[1] A. Eshraghian, C. D. Martin, and N. R. Morgenstem, "Movement Triggers and Mechanisms of two Earth Slides in the Thompson River Valley, British Columbia, Canada," Canadian Geotechnical Journal, vol. 45, pp. 1189-1209, 2008.

[2] P. S. Lin, J. Y. Lin, J. C. Hung, and M. D. Yang, "Assessing Debris-Flow Hazard in a Watershed in Taiwan," Engineering Geology, vol. 66, pp. 295-313, 2002.

[3] S. C. Hsu, J. Y. Lin, and M. D. Yang, "Slope Instability of High Terrace Deposits under Extreme Weather Conditions," Geo-Congress 2013, Geotechnical Special Publication 231, ASCE, pp. 462-471, 2013. 